\title{
SYSTÈME ET LOGICIELS POUR LA SURVEILLANCE DES PASSES À POISSONS.
}

\author{
M. CATTOEN (1), M. LARINIER (2) et N. THOMAS (3)
}

(1) INP-ENSEEIHT, 2 rue Camichel, BP 7122, 31071 Toulouse, France.

(2) CSP-CEMAGREF-GHAAPPE, Institut de Mécanique des Fluides, Avenue du Professeur Camille Soula, 31400 Toulouse, France.

(3) Université de La Rochelle, Avenue de Marillac, 17042 La Rochelle Cedex 1, France.

ieçu le 12 juillet 1999

iscepté le 19 juillet 1999
Received 12 July, 1999

Accepted 19 July, 1999

\section{PÉSUMÉ}

Le but de cet article est de présenter un système et les logiciels associés pour la surveillance des passes à poissons en vue du comptage et de l'identification des espèces. Le système proposé fonctionne en deux phases: une phase d'enregistrement sur le site d'images numérisées et comprimées et une phase de dépouillement interactif des enregistrements. Dans certaines conditions, il est possible d'effectuer un dépouillement automatique qui met en œuvre un processus de suivi pour accomplir le comptage et un processus de reconnaissance des espèces basé sur le calcul de caractéristiques géométriques pertinentes. Le système a été installé et testé sur deux sites : la passe à bassins successifs du Bazacle sur la Garonne et l'ascenseur de Tuilières sur la Dordogne.

Mots-clés : poisson migrateur, passe à poissons, contrôle, analyse d'image.

\section{SYSTEM AND RELATED SOFTWARE FOR MONITORING FISH PASSES.}

\section{ABSTRACT}

The aim of this paper is to describe a system and its related software for monitoring fish passes (species identification and counting). The proposed system works in two stages : the first one involves recording of digitized and compressed images, the second consists of interactive reviewing of recorded images. The reviewing may be automated under certain conditions. In this case, it uses a tracking process for counting and a species recognition process based on discriminant shape features extraction. The system has been installed and tested at Le Bazacle pool fish pass on the Garonne river and at the Tuilières fish lift on the Dordogne river.

Key-words : migratory fish, fish passage, monitoring, image analysis. 


\section{INTRODUCTION}

Dans le cadre des plans de restauration des populations de poissons migrateurs qiil se sont développés au cours de ces quinze dernières années en France, on a assisté l'aménagement de nombreuses passes à poissons visant à rouvrir les voies de migratior. Des stations de contrôle, destinées à la fois à s'assurer de l'efficacité des dispositifs et suivre les flux et l'évolution des stocks des espèces migratrices concernées par le; mesures de restauration, ont été installées sur la plupart des grands axes de migration.

Les parties amont des ouvrages de franchissement ont été aménagées pour permettre l'observation et le comptage par visualisation latérale à travers une vitre, de: poissons qui les franchissent. La technique la plus fréquemment utilisée est celle de ha vidéo-surveillance : le système, dénommé CERBERE (TRAVADE, 1990), comprend une caméra vidéo reliée à un magnétoscope à temps échelonné enregistrant à vitesse réduit et se mettant à vitesse normale sur une alarme extérieure. Cette alarme est générée per? un analyseur d'image lors de l'intrusion d'un poisson dans le champ d'observation. Cette technique nécessite le dépouillement manuel en différé des bandes vidéo par un opérateur.

Ce travail de dépouillement demeure néanmoins fastidieux: il peut représenter environ $1 \%$ du temps réel de surveillance pour les très faibles passages (quelques dizaines d'individus par jour), de $4 \%$ à $10 \%$ du temps réel pour les faibles passages (moins de 400 poissons/jour), et de $15 \%$ à $20 \%$ du temps réel pour des passages intenses (3 000 à 5000 poissons/jour) (TRAVADE et LARINIER, 1992). Des recherches ont été entreprises depuis plusieurs années pour développer un système visant à simplifier et à alléger ces opérations de dépouillement, et dans certains cas bien particuliers, à envisager leur automatisation (CASTIGNOLLES, 1995).

Le système développé fonctionne en deux phases. La première est une phase d'enregistrement sur le site de séquences d'images numérisées et comprimées. La deuxième est un dépouillement interactif des enregistrements en temps différé. La possibilité d'un dépouillement automatique a été étudiée. Dans ce dernier cas, le logiciel inclut un processus de suivi sur une série d'images pour accomplir le comptage, et un processus de reconnaissance d'espèces basé sur l'extraction de caractéristiques géométriques pertinentes.

Le système a été installé dans les passes à poissons des usines de Tuilières sur la Dordogne et du Bazacle sur la Garonne à Toulouse. Sur ces deux sites, les principales espèces concernées sont, pour les migrateurs amphibiotiques, le saumon, la truite de mer, l'alose, la lamproie marine et l'anguille, et pour les espèces holobiotiques le barbeau, la brème et le gardon (TRAVADE et al., 1998).

On décrira dans la suite le système d'acquisition des images, le logiciel d'enregistrement des séquences d'images, ainsi que les logiciels de dépouillements interactifs et automatiques. Les résultats et les limites actuelles de la technique ainsi que l'évolution probable du système dans les prochaines années seront présentés.

\section{ACQUISITION DES IMAGES}

Dans la plupart des passes à poissons, la zone d'observation est située dans un rétrécissement d'une largeur de l'ordre de $0,40 \mathrm{~m}$ à $0,50 \mathrm{~m}$ au droit d'une baie vitrée. Pour que le comptage se fasse dans de bonnes conditions, il est nécessaire que les poissons ne stationnent pas devant la vitre et qu'ils effectuent le moins possible d'allers et retours. A cet effet, des vitesses d'écoulement suffisantes sont maintenues dans la zone 
"̈observation. On limite d'autre part la formation de zones de décollement au niveau de la vitre susceptibles de provoquer la stagnation des poissons, par l'installation de convergents à l'amont immédiat de la partie rétrécie située au droit de la vitre de comptage.

La visualisation des poissons est améliorée par la mise en place sur la paroi arrière, sit d'une plaque de couleur claire carroyée, soit d'un système de rétro-éclairage consistant en un caisson ou local étanche contenant un éclairage et fermé à l'avant par un r atériau translucide qui diffuse la lumière. Dans le premier cas, un éclairage de la zone de 1.sualisation s'impose pour maintenir des conditions suffisantes d'éclairement de jour (.) mme de nuit.

Un éclairage progressif de la passe à poissons (généralement avec des lampes à lapeur de mercure) de part et d'autre de la section de comptage est assuré de façon à e iter une transition trop brutale lumière-obscurité susceptible d'induire un blocage des foissons.

Dans la passe à poissons de Tuilières, le principe du rétro-éclairage a été adopté. II fermet d'obtenir des images bien contrastées où les poissons apparaissent comme des silhouettes sombres sur un fond blanc. Ce choix sera justifié ultérieurement, pincipalement par les contraintes des procédés d'enregistrement (simplicité et performance des techniques de compression) et des méthodes de traitement d'images en vue de l'extraction des caractéristiques de forme des poissons. La Figure 1 présente la vue de dessus de la passe à poissons au niveau de la vitre d'observation schématisant les conditions d'acquisition des images sur le site de Tuilières.

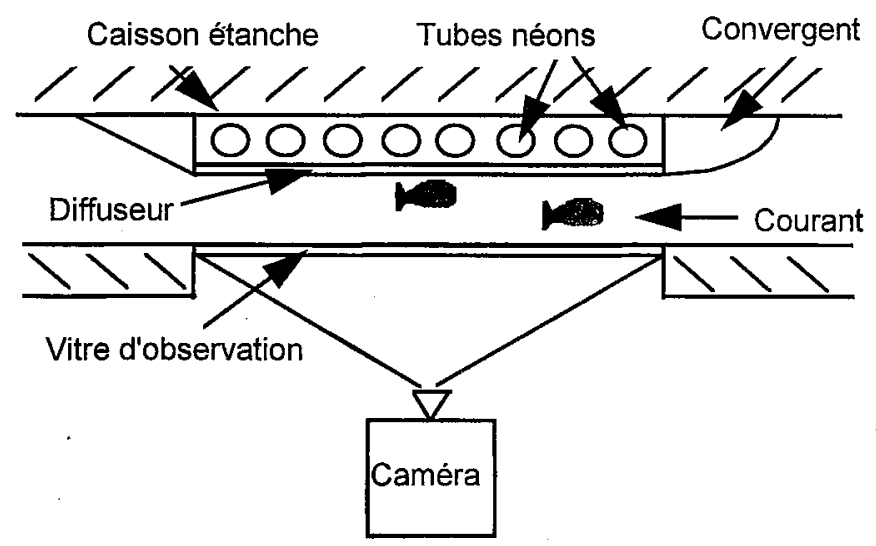

Figure 1

Conditions d'acquisition des images sur le site de Tuilières.

\section{Figure 1}

\section{Acquisition conditions of images at Tuilières.}

La configuration de la passe à poissons du Bazacle ne permet pas d'utiliser cette solution de rétro-éclairage: la réduction de section provoquée par l'installation d'un caisson lumineux entraînerait en effet, une augmentation de la vitesse d'écoulement incompatible avec les capacités de nage des espèces de petite taille.

La chaîne d'acquisition des images utilisée dans les deux sites comprend les éléments suivants :

- un micro-ordinateur de type PC muni d'une carte de numérisation d'images, elle-même reliée à une caméra vidéo N/B ; 
- un moniteur de visualisation pour le contrôle des images vidéo ;

- un ensemble de deux lecteurs-enregistreurs de disques numériques amovibles ;

- un onduleur qui alimente l'unité centrale et les lecteurs-enregistreurs.

La chaîne d'acquisition est représentée sur la Figure 2.

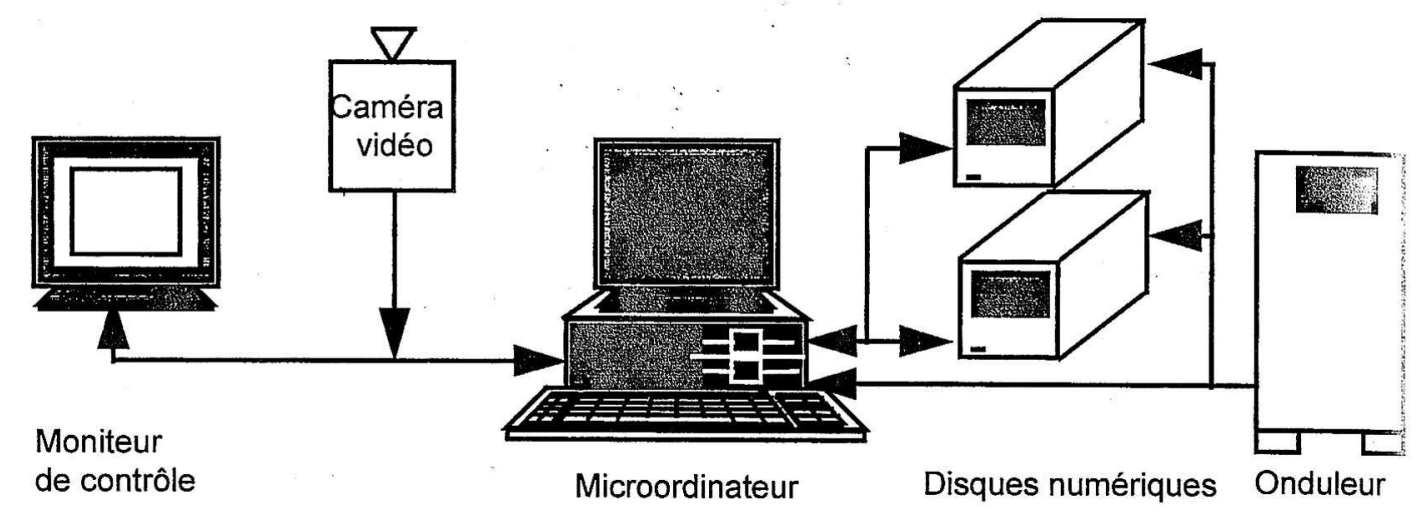

Figure 2

Chaine d'acquisition.

Figure 2

Acquisition system.

Les Figures 3 et 4 représentent des exemples d'images numérisées sur le site du Bazacle et comprimées en mode JPEG avec un taux de compression d'environ 25.

\section{Figure 3}

Image comprimée (alose) à la station de contrôle du Bazacle.

\section{Figure 3}

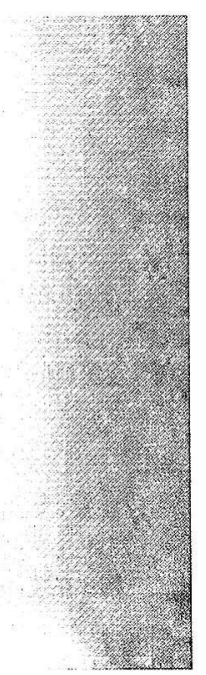

Compressed image (allis shad) at Le Bazacle counting station.

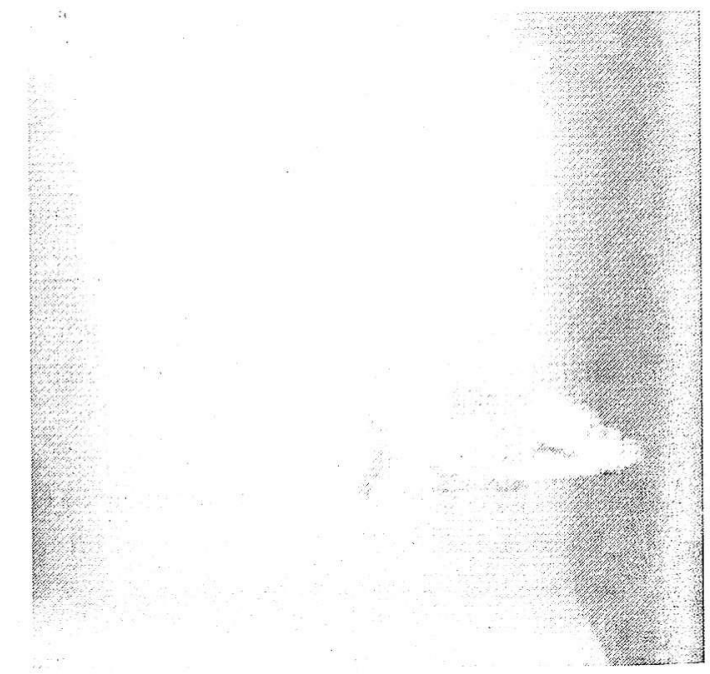

Figure 4

Image comprimée (brème) à la station de contrôle du Bazacle.

Figure 4

Compressed image (bream) at Le Bazacle counting station. 


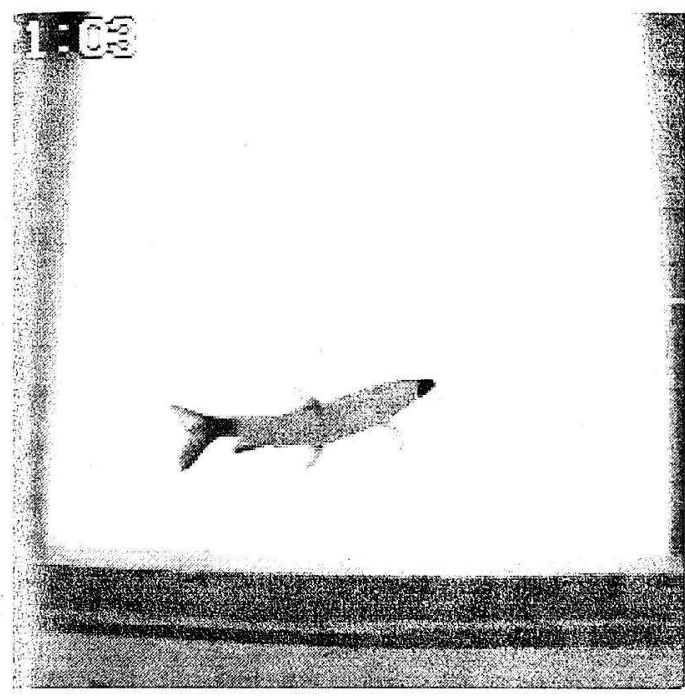

igure 5

Image N/B à la station de contrôle de Tuilières.

\section{Figure 5}

B/W image at Tuilières counting station.

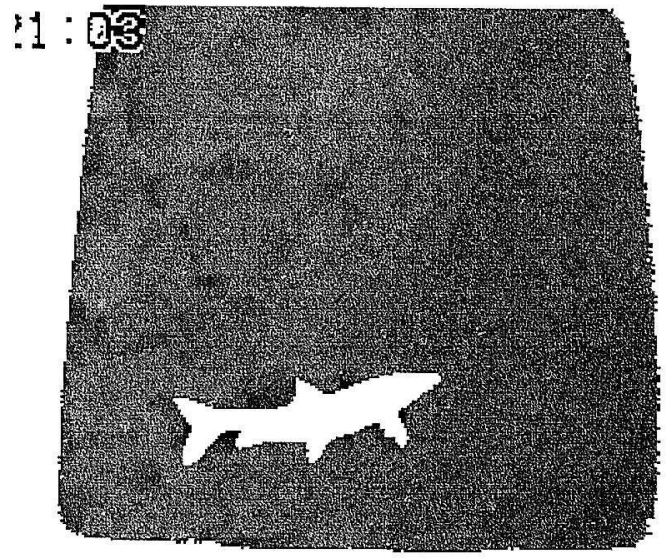

Figure 6

Image binarisée à la station de contrôle de Tuilières.

Figure 6

Binarized image at Tuilières counting station.

La Figure 5 représente un exemple d'image numérisée sur le site de Tuilières et la Figure 6 montre l'image binaire correspondante (seuillée et inversée).

\section{LOGICIEL D'ENREGISTREMENT DE SÉQUENCES}

Ce logiciel permet l'enregistrement de séquences selon deux modes: enregistrement déclenché ou enregistrement continu.

Les images peuvent être comprimées selon deux procédés : compression JPEG pour les images N/B et compression par « codage par segments » pour les images binaires (CATTOEN, 1994).

Dans le premier mode, seules les images remplissant des conditions d'alarme (mouvement par exemple) sont enregistrées.

Dans le second mode, les images sont enregistrées en permanence à vitesse constante ; ici, seule la compression sur des images binaires est utilisée.

Le logiciel utilise un certain nombre de paramètres que l'on doit fixer avant de lancer l'enregistrement ; ces paramètres sont stockés dans un fichier de configuration mis à jour automatiquement à chaque modification d'un paramètre (Figure 7 ).

L'enregistrement s'effectue sans interruption : lorsqu'un disque est plein, l'autre est automatiquement sélectionné ; le disque plein doit alors être remplacé.

Si l'enregistrement est arrêté (volontairement ou lors d'une coupure d'électricité), les paramètres courants sont sauvegardés dans le fichier de configuration. Lors de la reprise, ils sont restitués pour déterminer les conditions initiales d'enregistrement. 
Dans le cas des images binaires, on peut définir sur l'image une fenêtre de taille ajustable pour éliminer les parties sombres fixes qui tendent à diminuer le taux de compression (paramètres: $x \min , x \max , y$ min et ymax). Si l'éclairage est non uniforme, it est possible d'utiliser un seuillage dynamique basé sur la mémorisation d'une image prise en l'absence de poisson (paramètre : mode seuil).

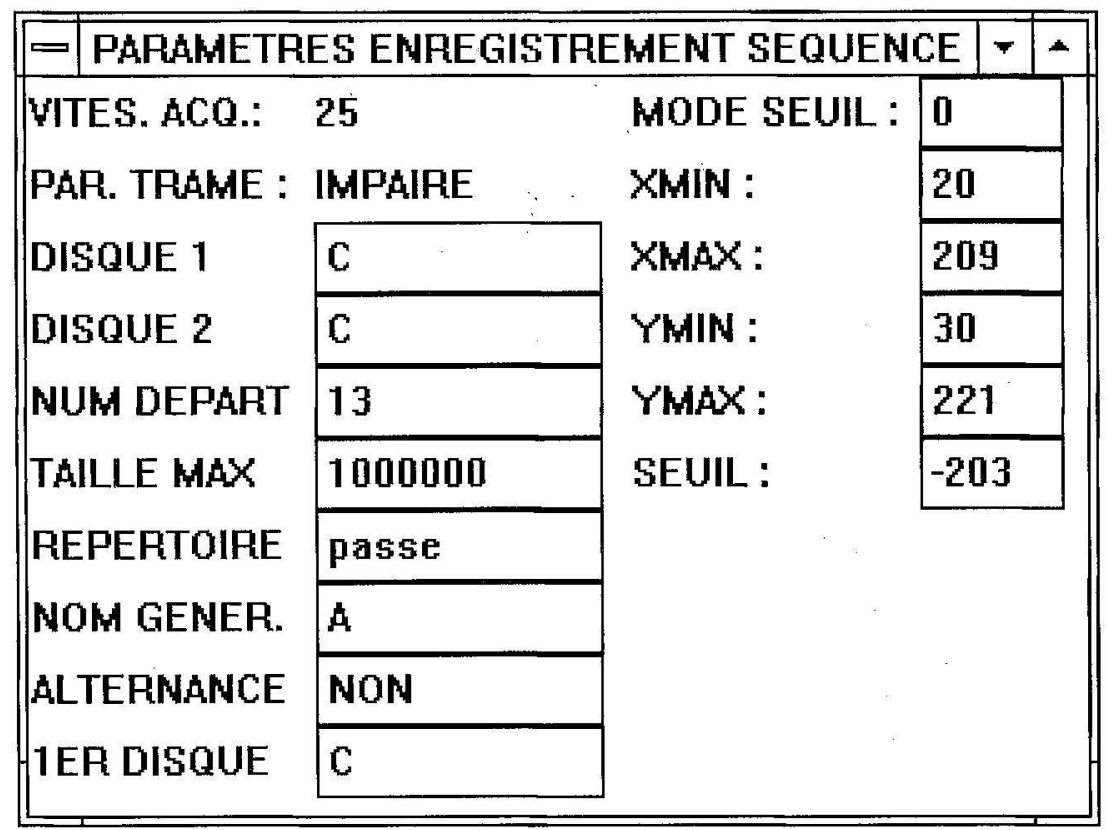

Figure 7

Paramètres d'enregistrement des séquences.

\section{Figure 7}

Recording parameters of sequences.

L'aspect de l'écran lors de l'enregistrement déclenché est présenté sur la Figure 8.

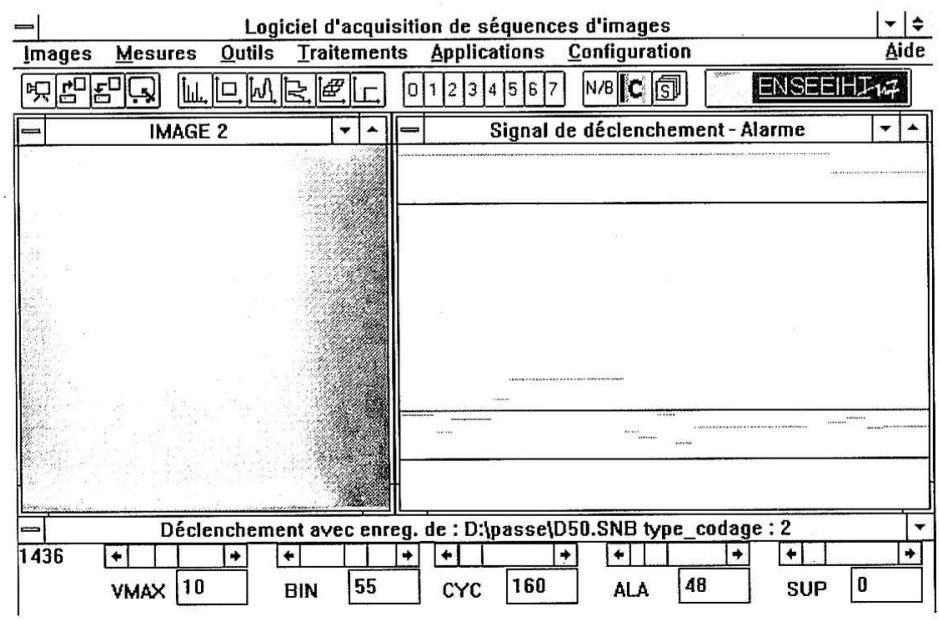

Figure 8

Ecran pour l'enregistrement déclenché.

Figure 8

Screen during triggered recording. 
L'enregistrement déclenché est soumis aux conditions d'alarme : modifications dans es images consécutives à des mouvements (présence de poissons ou d'objets divers: ranches, feuilles...).

L'image est découpée en zones de dimension et position ajustables nommées fenêtres" (Figure 9). Le principe de la détection de mouvements consiste à calculer ériodiquement la valeur moyenne de la luminance de chaque fenêtre, à prendre le naximum des différences absolues et à comparer ce maximum à un seuil ajustable. orsque ce seuil est dépassé, une alarme est déclenchée pour une durée variable. Durant activation de l'alarme, les images sont enregistrées.

La Figure 10 montre un exemple de l'évolution du signal de déclenchement et du ignal d'alarme en fonction du temps.

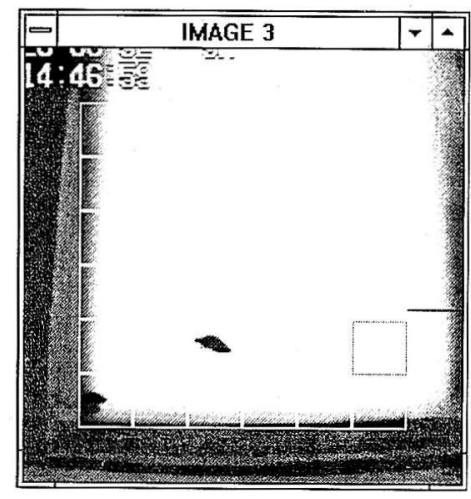

Figure 9

Découpage de l'image en fenêtres.

Figure 9

Splitting image into windows.

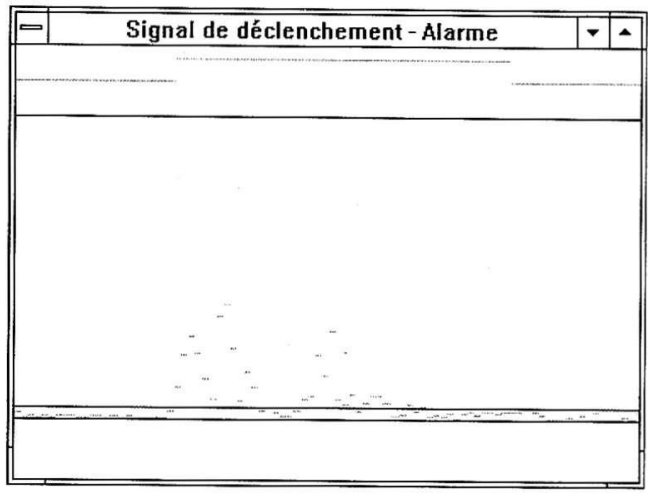

Figure 10

Signal de déclenchement et alarme.

Figure 10

Triggering and alarm system.

On peut régler les paramètres de déclenchement suivants :

- cycle de mesure (période entre deux comparaisons d'images successives) ;

- seuil de déclenchement de l'alarme;

- seuil de « suppression » qui prend en compte les variations globales sur la zone surveillée ;

- temporisation de l'alarme (temps de maintien d'alarme après dépassement du seuil) ;

- nombre d'images précédant la condition de déclenchement qui doit être enregistré.

La fonction d'édition de fenêtres permet:

- d'ajouter de nouvelles fenêtres ;

- de supprimer des fenêtres ;

- de modifier la taille et la position des fenêtres ; 
- d'ajuster la sensibilité de chaque fenêtre ;

- d'activer ou de désactiver des fenêtres.

Tout comme les paramètres d'enregistrement, les paramètres de déclenchemer sont stockés dans un fichier de configuration mis à jour automatiquement après chaqu modification d'un paramètre.

L'aspect de l'écran lors de l'enregistrement continu est présenté sur la Figure 11.

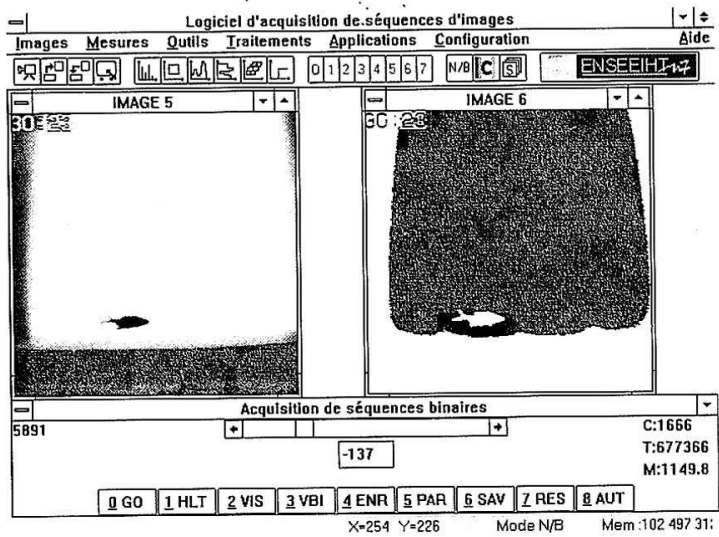

Figure 11

Ecran pour l'enregistrement continu.

\section{Figure 11}

Screen during continuous recording.

\section{LOGICIEL DE DÉPOUILLEMENT INTERACTIF}

Ce logiciel peut travailler à partir de séquences déclenchées ou continues obtenue grâce au logiciel d'enregistrement.

La Figure 12 montre l'aspect de l'écran après sélection d'une séquence déclenchét

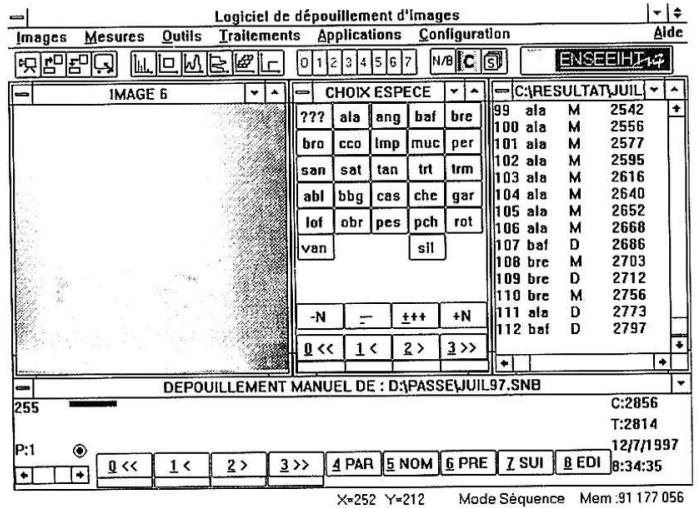

\section{Figure 12}

Ecran pour le dépouillement interactif.

\section{Figure 12}

Screen during interactive reviewing. 
On y distingue :

- une fenêtre affichant les images de la séquence ;

- une fenêtre affichant:

* une barre d'avancement matérialisant la position de l'image courante dans la équence ;

* des paramètres de l'image courante :

- numéro ;

- nombre de codes ;

- datation.

* un menu de sélection comprenant les options suivantes :

- 0 : Recul en animation;

- 1 : Recul image par image ;

- 2 : Avance image par image;

- 3 : Avance en animation ;

- 4: Paramètres du fichier;

- 5 : Choix d'un nouveau fichier ;

- 6 : Fichier de numéro précédent ;

- 7 : Fichier de numéro suivant ;

- 8 : Edition pour constituer une séquence à partir de morceaux de séquences.

En positionnant le curseur commandé par la souris dans la zone sous la barre d'avancement et en cliquant sur le bouton gauche, on peut se déplacer dans la séquence et atteindre, avec plus ou moins de précision selon la longueur de la séquence, une image particulière.

Dans le cas des enregistrements binaires continus, un diagramme représentant par des raies verticales de différentes hauteurs les surfaces (nombre de points blancs) des images de la séquence, est rajouté sous la barre de défilement. II présente l'intérêt de mettre en évidence de façon globale pour l'utilisateur, la présence d'objets dans la scène: qu'il peut alors visualiser très rapidement. De plus, une fonction d'affichage des images selon un critère de surface minimum permet d'éliminer de la visualisation les images vides ou contenant des objets de petite taille.

Nous appelons "événement ", la sortie d'un poisson de la zone d'observation. A chaque événement sont associées les informations suivantes stockées dans un fichier de résultats : espèce, montée ou descente, numéro de l'image de sortie, date et heure.

Deux fenêtres sont utilisées pour le dépouillement :

- une fenêtre permettant le choix des espèces et du sens de passage pour chaque événement, ainsi que le contrôle de certaines commandes d'animation ;

- une fenêtre où est visualisée la liste des événements enregistrés (contenu du fichier de résultats) (Figure 13). A partir de cette fenêtre, on peut :

* accéder directement à l'événement associé à une ligne (animation des 25 images précédant l'événement) ; 
* éditer une ligne: modification des paramètres (espèce, montée ou descent numéro de l'image de sortie et effacement).

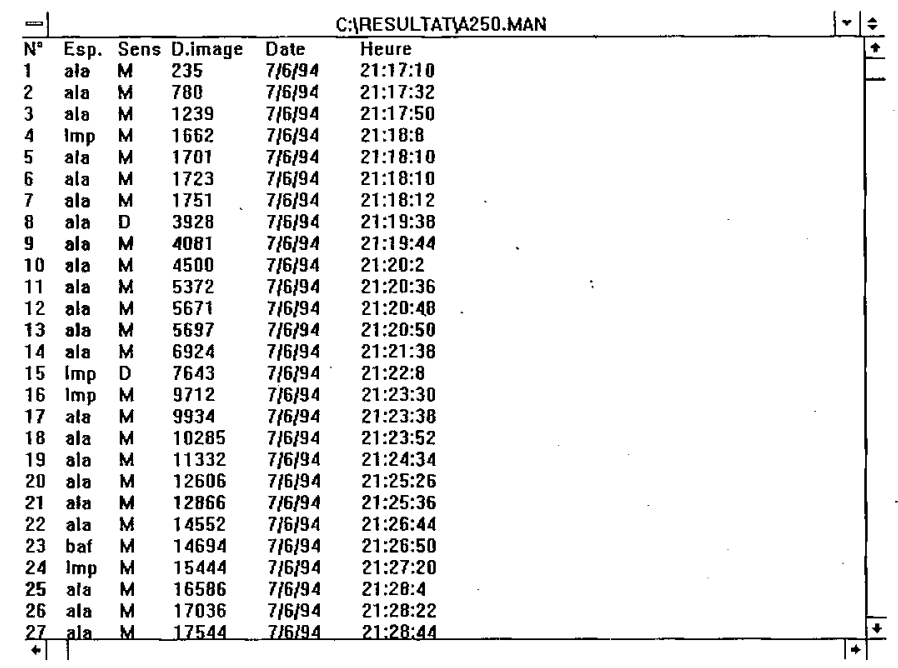

\section{Figure 13}

\section{Contenu d'un fichier de résultats.}

\section{Figure 13}

\section{Content of a results file.}

\section{LOGICIEL DE DÉPOUILLEMENT AUTOMATIQUE}

Ce logiciel ne travaille que sur les séquences binaires continues. Les deux fonctiol principales sont le comptage et l'identification des espèces (CASTIGNOLLES et al, 1995

Un poisson doit être compté lorsqu'il traverse la passe à poissons d'aval en amo et décompté dans le cas contraire. II ne doit pas être compté s'il fait demi-tour. Pour cel un processus de suivi de chaque poisson est mis en œuvre dès qu'il entre dans le chan d'observation et jusqu'à ce qu'il en sorte. Le suivi s'accomplit sur son centre de gravité ‘ utilisant une fenêtre de poursuite. Pour que cette opération s'effectue correctement, il fa que la vitesse d'acquisition soit constante et de valeur élevée (25 images/seconde), car it déplacements peuvent être très rapides. D'autre part, il est nécessaire que le poissı puisse être extrait automatiquement du fond par seuillage, ce que permet le rétro-éclaira! qui le fait apparaître en silhouette.

La méthode de reconnaissance utilise un processus préalable d'apprentissage c consiste à construire une base de données comportant les caractéristiques géométriqu de formes mesurées sur un certain nombre de poissons identifiés visuellement par I opérateur. Une classe est alors affectée à chaque espèce. La base de données $\epsilon$ actuellement limitée à 14 espèces: alose, anguille, barbeau, brème, brochet, carf lamproie, muge, perche, sandre, saumon, tanche, truite et truite de mer.

A chaque image, les caractéristiques géométriques sont calculées sur chaque ob. à reconnaître pour être comparées à celles de la base de données. Notons qu'il s'a d'une reconnaissance globale sur la forme et non d'une reconnaissance sur des parties । la forme (nageoires, tête,...). Ceci implique que le processus de reconnaissance ne d pas être réalisé sur un objet ayant un contact avec l'un des bords de l'image, car dans cas il peut être tronqué. 
Lorsqu'il sort du champ d'observation, un poisson donné présente des résultats de reconnaissance qui peuvent être différents selon les images de la traversée. Un vote majoritaire avec seuil de décision est alors utilisé pour décider de son espèce ( reconnaissance dynamique "). Ce seuil correspond au pourcentage minimal d'images de la traversée qui doit donner le même résultat de reconnaissance pour autoriser la décision. Si ce seuil n'est pas atteint, le poisson est considéré comme « non identifié ». séquence.

La Figure 14 présente l'aspect de l'écran après le dépouillement automatique d'une

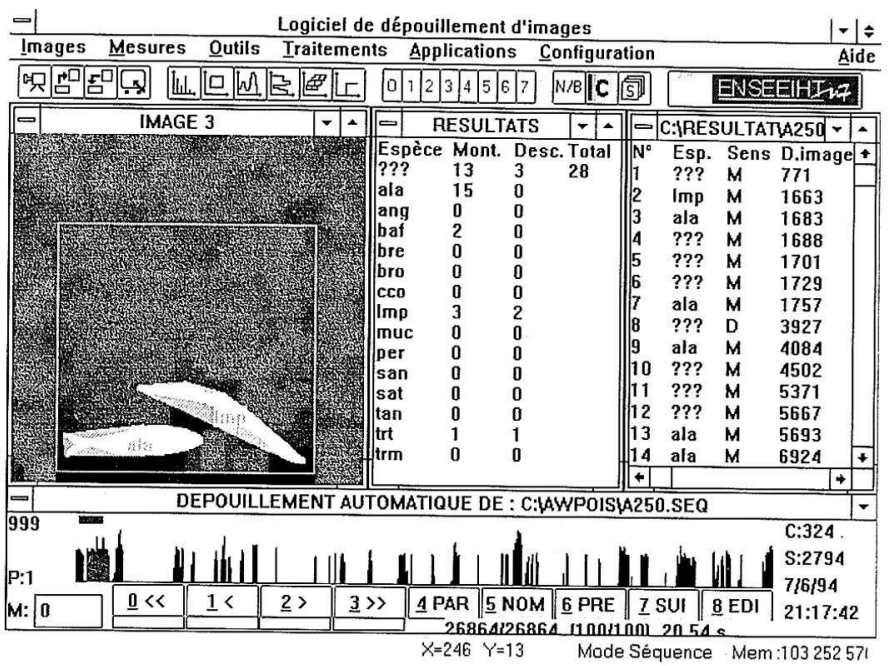

Figure 14

Ecran pour le dépouillement automatique.

Figure 14

Screen during automatic reviewing.

Deux fenêtres sont utilisées pour la visualisation des résultats:

- une fenêtre présentant le bilan global des passages;

- une fenêtre où est visualisée la liste des événements enregistrés (fichier de résultats de structure identique à celui obtenu interactivement) (Figure 13). Les mêmes fonctions que celles du dépouillement interactif sont disponibles (animation d'un événement, édition).

D'autre part, la fenêtre de choix des espèces et du sens de passage utilisée pour le dépouillement interactif est également disponible afin de permettre l'ajout éventuel des événements non détectés par le dépouillement automatique.

\section{RÉSULTATS}

Sur le site du Bazacle, le système d'enregistrement déclenché a été utilisé pendant une durée d'un an en parallèle avec le système CERBERE en place. Les résultats des comptages et les temps de dépouillement ont été comparés sur une durée d'un peu plus de 6 mois incluant la période de migration des aloses, période pendant laquelle environ 10000 poissons ont été comptabilisés. 
Sur cette période, le volume de stockage représente environ 1,8 Giga-octets, ce qu: correspond à des volumes moyens de 8,5 Méga-octets/jour et 1,8 Méga-octets/poissor. avec une vitesse d'enregistrement de 9 images/seconde.

En période de très faible migration, le volume d'enregistrement peut descendr: au-dessous de 0,2 Méga-octets/jour, alors qu'en période de migration d'alose, il passe e: moyenne à 64 Méga-octets/jour. Des valeurs beaucoup plus importantes (plus d 100 Méga-octets/heure) peuvent être atteintes transitoirement: ces pointes $n$ : correspondent pas à des passages de poissons, mais à des alarmes quasi-permanente: induites par des abaissements intempestifs du niveau d'eau.

Par rapport au système CERBERE, les temps de dépouillement sor: considérablement réduits. Pour la période considérée de 6 mois 18 jours, le temps d: dépouillement a été réduit d'un facteur 8 : le temps de dépouillement a été de $11 \mathrm{~h} 20 \mathrm{mi}:$ (soit environ 3,5 min/jour) avec le système développé et de $90 \mathrm{~h} 11 \mathrm{~min}$ (soit enviro: $27 \mathrm{~min} / \mathrm{jour}$ ) avec le système CERBERE. Ce facteur varie cependant en fonction $\mathrm{d}$ : nombre de poissons transitant dans l'ouvrage : en période de très faible passage (moin: de 10 individus/jour), il varie de 20 à plus de 50, alors qu'il tend vers 1 pour des passage: supérieurs à 1000 poissons/jour (Figure 15).

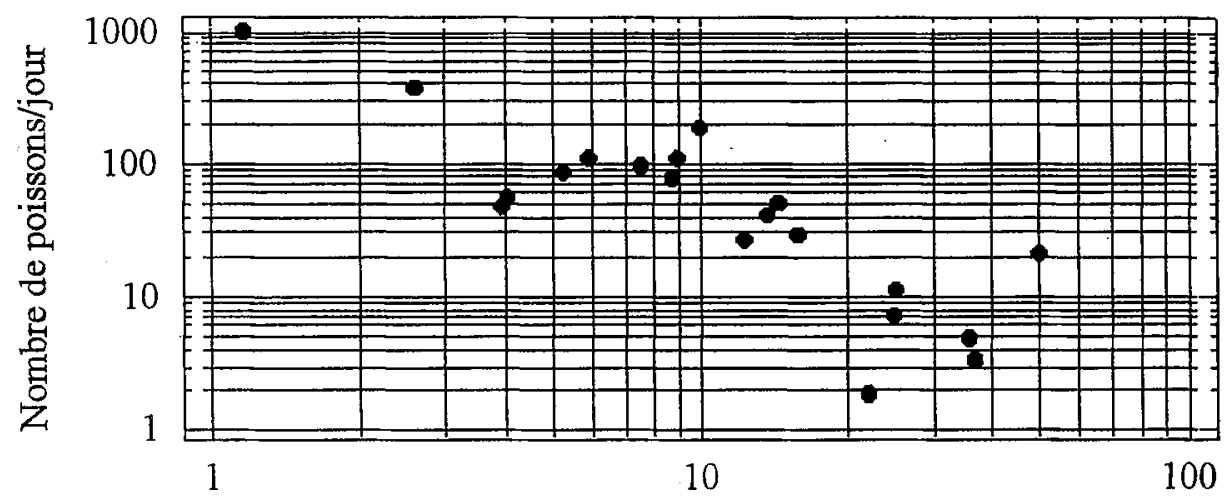

Rapport des durées de dépouillement (vidéo/analyse image)

\section{Figure 15}

\section{Comparaison des temps de dépouillement entre la vidéo et le système développé.}

\section{Figure 15}

\section{Comparison of reviewing times between the developped system and CERBERE system.}

Ce gain se fait néanmoins avec une certaine perte d'efficacité, en particulier sur les poissons de petite taille, tout au moins avec les réglages effectués lors de la comparaison Globalement le nombre de poissons compté par le système a été de $88 \%$ de celui compt par le système CERBERE. La différence sur les poissons de grande taille comme les salmonidés grands migrateurs est cependant minime : le pourcentage de poissons compte par le système atteint $98 \%$, un seul individu sur une cinquantaine n'ayant pas été détecté Pour les aloses, ce pourcentage est voisin de $94 \%$ (4 290 contre 4553 individus).

Sur le site de Tuilières où les conditions d'acquisition sont favorables (rétro-éclairage, peu de bulles, transparence de l'eau élevée,...), les modes d'enregistrement déclenché N/B ou d'enregistrement continu d'images binaires peuven s'utiliser indifféremment. Le mode d'enregistrement déclenché N/B a été utilisé en parallèlt avec le système CERBERE en place et testé sur des périodes caractéristiques. 
Sur une durée de plus de 4,5 mois incluant la période de migration d'alose, période pendant laquelle environ 50000 poissons ont été comptabilisés, le volume de stockage représente environ 55 Giga-octets, ce qui représente une capacité de stockage moyenne de 40 Méga-octets/jour et en moyenne 1,1 Méga-octets/poisson, avec une vitesse d'enregistrement de 9 images/seconde. En période de pic de migration (alose et lamproie), e volume de stockage peut dépasser 300 Méga-octets/jour, alors qu'en période de très aible migration il peut descendre à 5 Méga-octets/jour.

Par rapport au système CERBERE, les temps de dépouillement sont d'autant plus éduits que le nombre de poissons est faible : le facteur de réduction est de 20 à 30 en jériode de faible activité migratoire alors qu'il devient inférieur à 3 en période de migration les aloses et peut atteindre 1 en période de migration des lamproies où les allers et etours au niveau de la vitre deviennent extrêmement fréquents.

Le mode d'enregistrement continu d'images binaires à une vitesse de 25 images/seconde a été utilisé sur une période de 2 mois incluant les migrations d'aloses. In a constaté une durée moyenne de stockage d'environ 4 jours sur un disque optique de 128 Méga-octets (volume moyen de stockage de 32 Méga-octets/jour), la durée minimale jbservée étant de 17 heures, la durée maximale de 8 jours. Une surveillance quotidienne sur le site a permis de gérer le changement des disques sans interruption d'enregistrement.

Sur un PC équipé d'un Pentium MMX à $200 \mathrm{MHz}$, la durée moyenne mesurée du dépouillement automatique est bien inférieure à la durée d'enregistrement (environ 80 fois). Le temps de traitement moyen par image en présence de poissons est de 5 millisecondes, mais il peut atteindre 50 millisecondes dans le cas d'un passage simultané d'un nombre important de poissons.

La principale difficulté rencontrée avec le dépouillement automatique réside d'une part dans l'impossibilité, même sur le site de Tuilières, d'acquérir des images de qualité suffisante et surtout constante pour permettre de fiabiliser la détection des silhouettes sans érosion des contours, d'autre part dans l'impossibilité de comptage lors des périodes de pic de migration en présence de recouvrements fréquents qui rendent rapidement inextricables le comptage et la reconnaissance. Cette reconnaissance reste d'autre part limitée à un nombre relativement restreint d'espèces caractéristiques par leur forme ou d'une taille suffisante. Elle devient illusoire pour des espèces de formes très voisines comme le saumon et la truite de mer.

\section{CONCLUSION}

Le système de surveillance de passes à poissons présenté ici est un système de dépouillement en temps différé d'images enregistrées sur site. Ce système et les logiciels associés présentent par rapport au système CERBERE, les avantages suivants:

- supports standards pour le stockage des images (disques fixes ou amovibles) ;

- accès rapide aux enregistrements et aux événements ;

- interfaçage aisé avec les tableurs et les logiciels de base de données pour l'exploitation des résultats ;

- réduction significative des temps de dépouillement. 
Ses fonctionnalités permettent de l'utiliser aussi bien dans les passes à poisson: non aménagées où l'enregistrement déclenché constitue un moyen efficace pou: minimișer le volume des données à stocker, que dans les passes à poisson rétro-éclairées où il autorise l'enregistrement continu binaire et donc le dépouillemerí automatique ultérieur.

On doit remarquer que dans les deux cas, les résultats sont fortement dépendant: d'un paramètre que l'on peut qualifier de critique.

Pour le premier, il s'agit du seuil de déclenchement : trop bas, il peut entraîner u': grand nombre de fausses alarmes, ce qui augmente le volume de données enregistrée: (saturation rapide des supports de stockage) et ralentit le processus de dépouillement: trop élevé, il peut provoquer le non-enregistrement de certains passages et partant, un sous-comptage.

Pour le second, il s'agit du seuil adopté pour la binarisation des images ; un mauvais; réglage peut conduire dans certains cas à un excès de données par prise en compte dé zones fixes sur l'image (saturation rapide des supports de stockage), dans d'autres cas à l'érosion ou à la dilatation des silhouettes de poissons, rendant difficile leur identification en mode interactif ou en mode automatique.

Concernant le logiciel d'enregistrement en mode déclenché, les travaux portent sur l'amélioration des algorithmes de déclenchement, afin d'éviter des enregistrements intempestifs provoqués par des baisses du niveau d'eau ou par la présence d'objets permanents dans la passe à poissons au niveau de la vitre (végétation aquatique par exemple).

Concernant le logiciel de dépouillement interactif, les travaux portent sur l'amélioration de l'ergonomie, afin d'augmenter encore le gain de temps par rapport au système CERBERE, plus particulièrement en période de passages intenses, où l'intérêt du système peut paraître moins évident.

Concernant le logiciel de dépouillement automatique, les travaux porteront sur la validation des résultats. La structure identique des fichiers de résultats obtenus interactivement et automatiquement facilite la comparaison des résultats issus des deux processus. Les algorithmes de comptage seront améliorés et la base de données d'apprentissage sera étendue à de nouvelles espèces. Une avancée significative sur ces deux points permettrait à plus long terme d'envisager les opérations de comptage et de reconnaissance automatiques en temps réel dans les sites où les conditions d'acquisition sont favorables et le nombre d'espèces est limité, ce qui allégerait notablement les contraintes de présence humaine. Néanmoins l'enregistrement des images reste la seule solution qui permet de contrôler a posteriori la validité des résultats obtenus et d'expliquer les causes de défaillances éventuelles.

\section{REMERCIEMENTS}

Nous tenons à remercier tout particulièrement $\mathrm{F}$. Travade pour sa collaboration dans le développement du système automatique. Nos remerciements vont également à O. Croze, J. Dartiguelongue et L. Carry qui ont effectué les tests sur les sites du Bazacle et de Tuilières. Nous sommes reconnaissants à la direction et au personnel technique des usines EDF du Bazacle et de Tuilières, en particulier à M. Bonnet qui a collaboré depuis le début à l'installation et au suivi du système à l'usine de Tuilières. Nous tenons enfin à remercier l'Institut National Polytechnique de Toulouse, le CEMAGREF, la Direction des 
Etudes et Recherches d'EDF, le Conseil Supérieur de la Pêche et la Région Midi-Pyrénées pour leur soutien technique ou financier.

\section{IBLIOGRAPHIE}

ZASTIGNOLLES N., 1995. Automatisation du comptage et de la reconnaissance des espèces dans les passes à poissons par l'analyse de séquences d'images, Thèse INP Toulouse, $172 \mathrm{p}$.

JASTIGNOLLES N., CATTOEN M., LARINIER M., 1995. Système de vision pour la surveillance des passes à poissons; $15^{\text {eme }}$ Colloque GRETSI, Juan-Les-Pins, 18-21 Septembre 1995, 1237-1240.

JATTOEN M., 1994. Enregistrer en continu des images numérisées en binaire, Rev. Electron, 42, 83-89.

RAVADE F., 1990. Monitoring techniques for fish passes recently used in France, Proc. Int. Symp. on fishways, Gifu, Japan, 119-126.

RAVADE F., LARINIER M., 1992. Les techniques de contrôle des passes à poissons (chapitre 12). Bull. Fr. Pêche Piscic., 326-327, 151-162.

IRAVADE F., LARINIER M., BOYER-BERNARD S., DARTIGUELONGUE J., 1998. Performance of four fish pass installations recently built on two rivers in south-west France. In : JUNGWIRTH M., SCHMUTZ S. and WEISS S. (Eds.), Chapter 11 in Fish migration and Fish bypasses, Fishing New Books, 146-170. 